\title{
Early Clinical Outcome of Comparative Study between Revision Operation and Radiofrequency Treatment for Management of Failed Back Surgery Syndrome
}

\author{
Se-Heum Park, Junseok W. Hur, Jang-Bo Lee, Jung Yul Park
}

Department of Neurosurgery, Korea University Anam Hospital, Seoul, Korea

Objective: Intractable back pain and radicular pain after lumbosacral spine surgery are challenges for surgeons because the pathophysiology of failed back surgery syndrome (FBSS) remains unknown. Various medications, exercise, reoperation, spinal cord stimulation, and various interventional treatments, such as epidural injection, and radiofrequency treatment, have been suggested as treatment options. However, the clinical outcomes for each treatment are unclear.

Methods: We retrospectively evaluated clinical outcomes of consecutive FBSS patients who underwent revision operation or radiofrequency treatment from 2014 to 2017, who previously showed response to nerve block. Pain was analyzed preoperatively and 1 month, 6 months, and 12 months postoperatively using a visual analogue scale (VAS). Short Form 36 (SF-36) health survey and Oswestry Disability Index (ODI) scores were assessed preoperatively and 12 months postoperatively.

Results: A total of 70 patients was included in the present study (33 males, 37 females). When comparing preoperative and postoperative 12-month results, back pain VAS score decreased from 5.5 to 4.2 for revision operation and from 5.1 to 4.7 for radiofrequency treatment. Leg pain VAS score decreased from 6.5 to 4.02 for revision operation and 6.2 to 4.3 for radiofrequency treatment. ODI score decreased from 70.9 to 36.1 for revision operation and 70.2 to 36.1 for radiofrequency treatment. SF-36 score increased from 28.9 to 64.1 for revision operation and from 29.6 to 59.6 for radiofrequency treatment. Differences between treatments were not statistically significant after 12 months $(p>0.05)$ but were at 1 month $(p=0.01)$. Conclusion: Compared with revision operation, radiofrequency treatment is not inferior on early clinical outcome (up to 1 year following the treatment) in terms of pain relief, functional capacity, patient satisfaction, and quality of life in patients with FBSS. Radiofrequency treatment can be considered in patients who are not good candidates for surgical treatment based on imaging results or underlying diseases and who are responsive to diagnostic/therapeutic nerve blocks.

Key Words: Failed back surgery syndrome; Radiofrequency ablation; Back pain

Received: July 12, 2021 Revised: July 22, 2021 Accepted: July 27, 2021

Corresponding Author: Jung Yul Park

Department of Neurosurgery, Korea University Anam Hospital, 73

Goryeodae-ro, Seongbuk-gu, Seoul 02841, Korea.

Tel: +82-2-920-5729, Fax: +82-2-929-0629,

E-mail: jypark98@korea.ac.kr

ORCID: https://orcid.org/0000-0002-7509-9485

\section{INTRODUCTION}

Failed back surgery syndrome (FBSS) is defined as surgical end-stage after one or several operative interventions on the lumbar neuroaxis, performed to relieve lower back pain, radicular pain, or the combination of both without positive out-

(C) 2021 Korean Neuro-Pain Society

(c) This is an Open Access article distributed under the terms of the Creative Commons Attribution Non-Commercial License (http://creativecommons.org/licenses/by-nc/4.0/) which permits unrestricted non-commercial use, distribution, and reproduction in any medium, provided the original work is properly cited. 
comes. Therefore, repeated surgery is not always the solution to back and leg pain. Understanding and explaining the mechanism of chronic pain can provide a solution [1]. FBSS is an inadequate term because it does not explain the principles of pain mechanism or pain from previous surgery [1]. Because FBSS is a restrictive denotation of blame and failure, the definition of FBSS has varied over time and remains controversial. Postoperative persistent syndrome, post-laminectomy syndrome, and chronic back and leg pain are other appropriate terms and do not contain the term "failure." Because FBSS is the most commonly used term in current research, FBSS was used as a term for persistent back and radicular pain after previous surgery in the present study [2].

Achieving total pain relief or the surgical goal can be difficult because complete pain relief is unrealistic. Explaining the differential diagnosis is important for treatment of postoperative symptoms. Foraminal stenosis, painful disc, pseudoarthrosis, neuropathic pain, recurrent herniated nucleus pulposus, iatrogenic instability, psychological factors, and infection are possible etiologies of postoperative symptoms [3].

FBSS is diagnosed after one or more operations are performed on the back at the same or adjacent segments, most often in the lumbar and sacral spine, and the incidence increases with the number of operations [4]. In a recent study, 5\% to $36 \%$ of patients who underwent lumbar spine surgery reportedly experienced FBSS [5].

Despite methodological problems in assessing surgical failure and success, many patients after lumbar surgery have chronic back pain and reduced health-related quality of life as well as high health care costs [5]. Appropriate management for patients with FBSS is difficult because clinical evaluation relies on subjective symptoms and precise evaluation of electromyogram, X-ray, and MRI. An established guideline for patients with back pain does not exist due to the diversity and complexity of the disease entity. A large number of multi-faceted studies are underway. Because various factors are associated with progression of chronic pain, treatment based on comparative analysis of multidisciplinary approaches in various fields is needed.

Patients with FBSS usually suffer mentally and physically from persistent back pain and chronic radiculopathy even after surgery, endoscopic surgery, spinal cord stimulation, physical therapy, epidural adhesiolysis, injection therapy, or oral medication [6]. Despite these efforts, the outcome of patient with FBSS often does not meet the presurgical expectation [7].

Surgical revision in FBSS patients has a low success rate and relatively high morbidity. However, revision operation should be considered in cases of bladder dysfunction, motor weakness, progressive neurological impairment of spinal cord injury, instability, and surgical hardware malfunction [5].

Radiofrequency (RF) treatment has advantage in terms of long-lasting pain relief, relative accurate targeting, and safety. The mechanism of radiofrequency treatment is based on the ability of a radiofrequency lesion to stop nociceptive input to the central nervous system without destructive effects on motor or sensory fibers. This can be achieved by using both heat producing therapeutic lesion making for medial branch of posterior primary rami making (for back pain) and neuromodulation treatment using non-destructive minimal heat treatment targeting on dorsal root ganglion (for leg pain and/ or paresthesia) [8].

Global reduction of evoked synaptic activity, structural change in blood-nerve barrier, fibroblast activation, and collagen deposition are other possible mechanisms of radiofrequency treatment. A minimally invasive, non-destructive procedure that is effective in treatment of chronic pain is appealing. Several study results shown that pain scores were significantly improved in the radiofrequency group at 1 year postoperatively compared with those in the placebo group. Consequent pain relief lead to decreased drug use and was associated with improved quality of daily life $[8,9]$. In the present study, the merits of revision operation and radiofrequency treatment for FBSS were assessed, and the outcomes and associated complications evaluated.

\section{MATERIALS AND METHODS}

\section{Patient Demographics}

Inpatient database from 2014 to 2017, was used to identify patients with FBSS. This retrospective cohort study included 313 patients diagnosed with FBSS. Inclusion criteria were duration of pain and follow-up period $>1$ year and back and leg pain visual analogue scale (VAS) score $>3$ on a 0 to 10 scale without persistent nerve root symptoms based on history, radiographic image, and physical examination. Subjects with specific radiologic findings, such as fracture, infection, neoplasm, acute lumbar disc herniation, and spondylolisthesis, were excluded ( $\mathrm{n}=243$ ). Thus, the present study included 70 patients (33 males and 37 females) with a mean age of 66 years who underwent revisional operation or radiofrequency treatment for FBSS. Preoperative MRI and X-ray were performed, and FBSS was diagnosed according to images and clinical findings.

After revision operation or radiofrequency treatment, some patients required additional surgery in 1 month or in 1 year. When intractable pain or motor weakness was found in pa- 
tients who received revision operation or radiofrequency treatment, additional surgery was necessary in patients who showed complications on radiographic imaging.

\section{Revision Operation}

The patients underwent various surgeries, including simple or multi-level decompressive laminectomy, flavectomy, discectomy, and instrumented fusion based on preoperative X-ray, MRI findings, and symptoms.

\section{Radiofrequency Treatment}

After patients were administered local anesthesia and placed in the prone position, radiofrequency devices were used to target the dorsal root ganglion and medial branch nerve under $\mathrm{C}$-arm guidance. A 10-cm, 22-gauge radiofrequency cannula with a $10-\mathrm{mm}$ active tip was introduced directly at the target point. Next, $50 \mathrm{~Hz}$ stimulation was applied to evoke paresthesia, and supplemental motor stimulation was administered at 2 $\mathrm{Hz}$ to ensure safety and correct probe position around the root. Pulsed dose radiofrequency therapeutic lesioning was performed as follows: $42^{\circ} \mathrm{C}$ for 4 minutes for dorsal root ganglion radiofrequency and $80^{\circ} \mathrm{C} 60$ seconds for medial branch radiofrequency. The electric field causes the movement of molecules that produces thermal energy, creating a small lesion that disrupts the pain signal. All patients undergoing radiofrequency treatment had a positive response to selective nerve and DRG block at the affected level. Radiofrequency treatment was repeated in patients who had a VAS score $>6$ at follow-up assessment (postoperative 6 months).

\section{Outcome Measures}

The VAS is a validated, subjective measurement scale rating from 0 (none) to 10 (worst) as a continuum between no pain and worst pain [10]. Oswestry Disability Index (ODI) measures disability on a scale ranging from 0 (no disability) to 100 (maximum disability possible) [11]. The 36-Item Short Form (SF-36) health survey questionnaire has eight scales: physical functioning $(\mathrm{PF})$, role physical (RP), bodily pain (BP), general health $(\mathrm{GH})$, vitality (VT), social functioning (SF), role emotional (RE), and mental health (MH). In component analyses, two distinct concepts are measured with the SF-36: a physical dimension, represented by the Physical Component Summary (PCS), and a mental dimension, represented by the Mental Component Summary (MCS). All scales contribute different proportions of scores for both PCS and MCS measures. All patients were evaluated before and 1, 6, and 12 months after treatment [12].

\section{Statistical Analysis}

All data are presented as mean \pm standard deviation. The chisquare test ( $\chi^{2}$ test) or Levene's test was used for equality of variances and Student's t-test for comparison of categorical variables in the two groups. Between-group comparisons of differences in VAS, ODI, and SF-36 scores before and after procedure based on treatment were analyzed using Levene's test for equality of variances and Student's t-test for continuous variables. All statistical analyses were performed using SPSS for Windows 21 . The results were considered statistically significant at a p-value $<0.05$.

\section{RESULTS}

\section{Demographics}

The mean age of the patients in the revision operation group was similar to that in the radiofrequency treatment group $(66.4 \pm 17.5$ years and $68 \pm 17.2$ years, respectively; $\mathrm{p}=0.71)$. The distribution of sex in the revision operation group was similar to that in the radiofrequency treatment group $(\mathrm{p}=0.97)$. Hypertension, diabetes, and BMI showed similar incidence in the revision operation and radiofrequency treatment groups $(\mathrm{p}=0.68, \mathrm{p}=0.28$, and $\mathrm{p}=0.65$, respectively). Distribution of muti-levels between revision operation and radiofrequency treatment was statistically similar ( $48 \%$ and $45 \%$, respectively; $\mathrm{p}=0.71)$.

The type of previous surgery was categorized simple decompressive laminectomy or discectomy, posterior lumbar interbody fusion. In total surgical group, the rate of patients who had fusion surgery did not differ much between the revision operation group and the RF treatment group (12\% and 19\%, respectively, $\mathrm{p}=0.77)$.

\section{VAS Score Results}

The pre-treatment back pain VAS score was similar between the revision operation and radiofrequency treatment groups $(5.51 \pm 15.5$ and $5.19 \pm 13.2$, respectively; $\mathrm{p}=0.36)$. The pretreatment leg pain VAS score was similar between the revision operation and radiofrequency treatment groups $(6.56 \pm 13.7$ and $6.29 \pm 12.1$, respectively; $p=0.38$ ); results are shown in Table 1.

In both groups, the average VAS scores obtained at 1,6 , and 12 months after treatment were significantly lower than the pre-treatment VAS scores (Fig. 1). Regarding the differentiation of pain intensity based on the back pain VAS score in the revision operation group, pain was aggravated at 1 month $(0.76 \pm 1.51)$. However, significant improvement was observed 
Table 1. Patient demographics

\begin{tabular}{|c|c|c|c|}
\hline & Revision operation $(n=39)$ & Radiofrequency treatment $(n=31)$ & p-value \\
\hline Age $^{*}$ & $66.4 \pm 17.5$ & $68 \pm 17.2$ & 0.71 \\
\hline $\operatorname{Sex}(M: F)(\%)$ & $18(46): 21(54)$ & $15(48): 16(52)$ & 0.97 \\
\hline Hypertension (\%) & $17(43)$ & $12(38)$ & 0.68 \\
\hline Diabetes (\%) & $12(30)$ & $10(32)$ & 0.28 \\
\hline $\mathrm{BMI}^{+}$ & $23.9 \pm 4.0$ & $24.1 \pm 4.8$ & 0.65 \\
\hline Previous surgery, multi-level (\%) & $19(48)$ & $14(45)$ & 0.71 \\
\hline Previous surgery, the number of fusion (\%) & $5(12)$ & $6(19)$ & 0.77 \\
\hline Pre VAS back ${ }^{*}$ & $5.51 \pm 15.5$ & $5.19 \pm 13.2$ & 0.36 \\
\hline Pre VAS leg* & $6.56 \pm 13.7$ & $6.29 \pm 12.1$ & 0.38 \\
\hline Pre SF $36^{*}$ & $28.9 \pm 12.0$ & $29.6 \pm 10.7$ & 0.79 \\
\hline Pre ODI* & $70.2 \pm 10.1$ & $70.9 \pm 13.2$ & 0.80 \\
\hline
\end{tabular}

The VAS ranges from 0 to 10, with lower scores indicating less severe symptoms. The SF-36 scores range from 0 to 100, with lower scores indicating less severe symptoms. The Oswestry Disability Index ranges from 0 to 100, with lower scores indicating less severe symptoms.

${ }^{*}$ Data are presented as mean \pm standard deviation.

'BMl: body mass index.

Table 2. Comparison of clinical outcome according to procedure

\begin{tabular}{lccc}
\hline & Revision operation & Radiofrequency treatment & p-value \\
\hline VAS back post procedure $\triangle$ pre-1 mo & $0.76 \pm 1.51$ & $-0.58 \pm 1.40$ & 0.01 \\
VAS back $\triangle$ pre-6 mo & $-0.69 \pm 1.48$ & $-0.54 \pm 1.43$ & 0.68 \\
VAS back $\triangle$ pre-12 mo & $-1.23 \pm 2.00$ & $-0.48 \pm 2.20$ & 0.14 \\
VAS leg $\triangle$ pre-1 mo & $-1.66 \pm 1.26$ & $-1.32 \pm 1.07$ & 0.23 \\
VAS leg $\triangle$ pre-6 mo & $-2.20 \pm 1.54$ & $-1.29 \pm 1.37$ & 0.10 \\
VAS leg $\triangle$ pre-12 mo & $-2.53 \pm 1.60$ & $-1.97 \pm 1.57$ & 0.20 \\
ODI $\triangle$ pre-12 mo & $-34.10 \pm 20.09$ & $-34.83 \pm 16.09$ & 0.24 \\
SF-36 $\triangle$ pre-12 mo & $35.12 \pm 21.38$ & $30.00 \pm 12.64$ & 0.86 \\
\hline
\end{tabular}

VAS back _ Revision operation _ Radiofrequency treatment

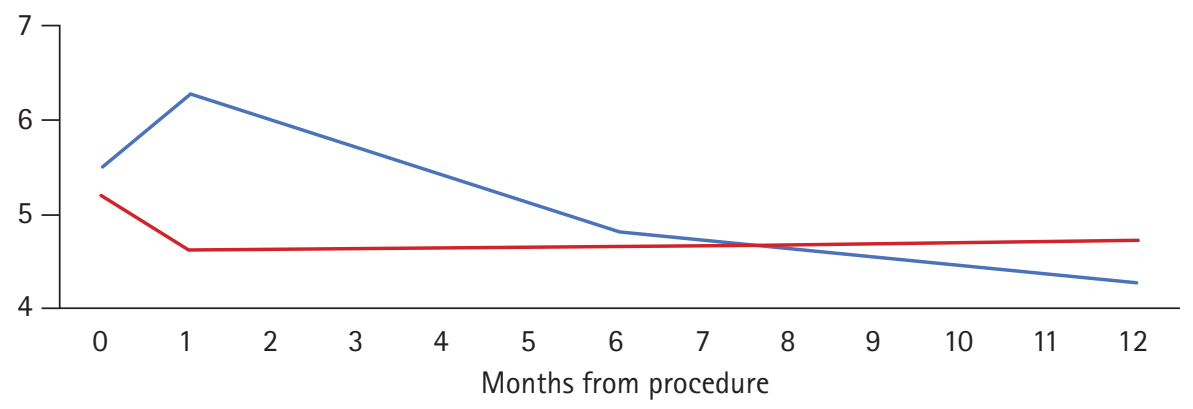

VAS leg Revision operation - Radiofrequency treatment

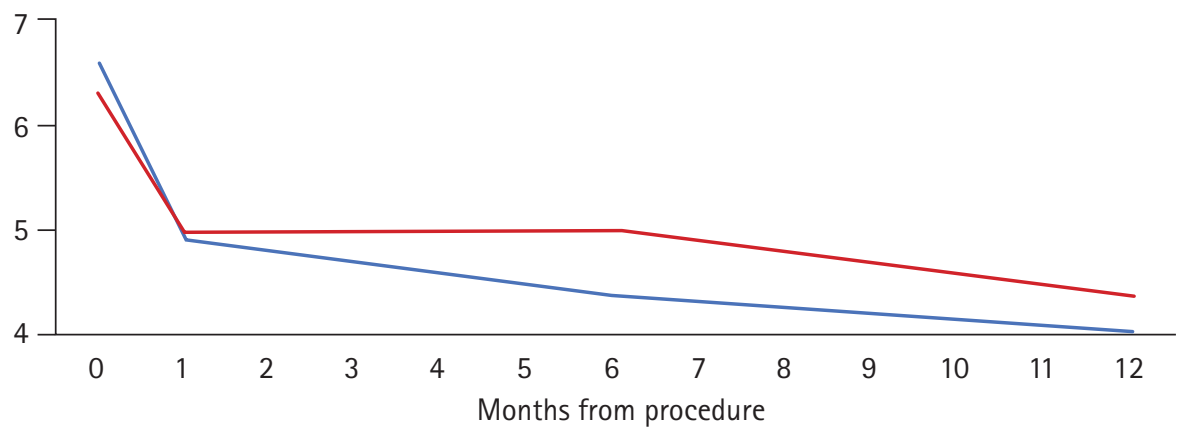

Fig. 1. VAS back and VAS leg months from procedure. 

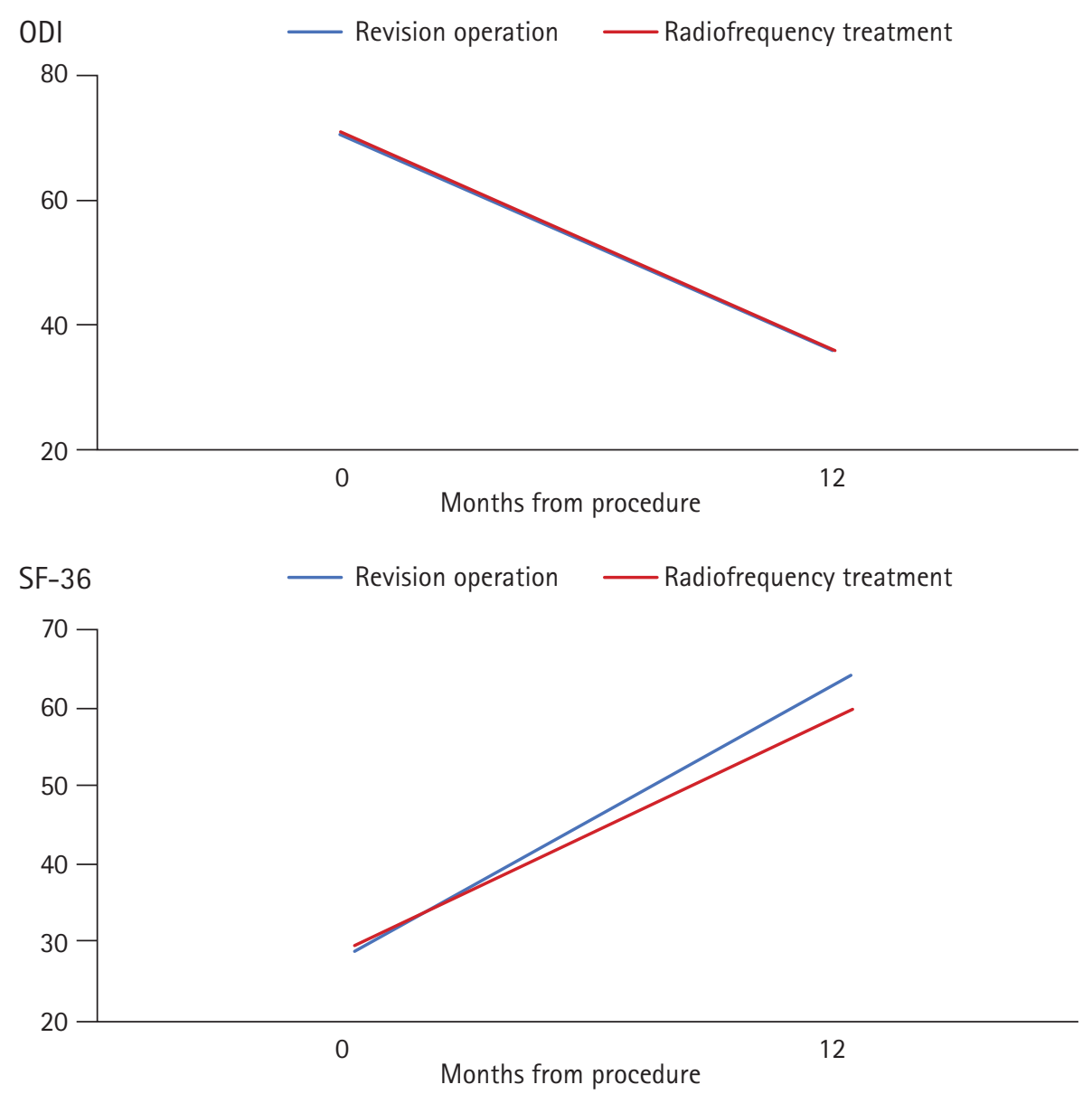

Fig. 2. ODI and SF-36 Months from procedure.

at 6 and 12 months $(-0.69 \pm 1.48$ and $-1.23 \pm 2.00$, respectively). A reduction of back pain VAS score from a mean of 5.5 to 4.2 was observed at 12 months in the revision operation group, and the mean score in the radiofrequency treatment group was reduced from 5.1 to 4.7; however, this was not a statistically significant difference between procedures $(p>0.05)$. A reduction of leg pain VAS score from a mean of 6.5 to 4.0 was observed at 12 months in the revision operation group, and the mean score in the radiofrequency treatment group was reduced from 6.2 to 4.3 but this was not a statistically significant difference $(\mathrm{p}>0.05)$ (Table 2).

\section{ODI and SF-36 Score Results}

The preoperative SF-36 and ODI scores were similar between the revision operation and radiofrequency treatment groups ( $\mathrm{p}=0.79$ and 0.80 , respectively) (Fig. 2). The ODI score at 12 months changed from a mean 70.2 to 36.1 in the revision operation group and from 70.9 to 36.1 in the radiofrequency treatment group, and the difference between procedures was not statistically significant $(p=0.24)$. A change in SF-36 score was observed at 12 months from a mean 28.9 to 64.1 in the revision operation group and from 29.6 to 59.6 in the radiofrequency treatment group, which was also not statistically significantly different $(p>0.05)$.

\section{Complications}

As shown in Table 3, wound dehiscence and deep infection were observed in the revision operation group. At 1 month, additional surgery was needed in one case due to screw malposition, producing immediate postoperative radiculopathy; the pain improved after additional surgery. At 1 year, additional surgery was needed in 2 cases due to adjacent segment disorder. One transient foot drop case occurred in the radiofrequency treatment group, and one patient suffered radiculopathy immediately after revision operation, and the symptom recovered after one month.

\section{DISCUSSION}

FBSS is a challenge for spine surgeons due to its multiple etiologies and lack of definitive treatment options, requiring multidisciplinary approaches [13]. Thorough imaging analysis, 
Table 3. Complication in patients according to procedure

\begin{tabular}{lcc}
\hline & Revision operation $(n=39)$ & Radiofrequency treatment $(n=31)$ \\
\hline Wound dehiscence & 1 & 0 \\
Deep infection & 4 & 0 \\
Requiring additional surgery in 1 mo & 1 & 0 \\
Requiring additional surgery in 1 yr & 2 & 0 \\
Neurological deficit & 1 & 1 \\
\hline
\end{tabular}

physical examination, and history taking are needed, and careful decision making is critical to determine benefits of reoperation. Rehabilitation and conventional medical therapy can aid in some patients with persistence of pain but may contribute to side effects and possible drug addiction, indicating the need for surgery or other treatment protocols (nerve blocks, radiofrequency treatment, spinal cord stimulation, and intrathecal drug pump, etc.) in refractory patients.

The main causes of FBSS in patients requiring reoperation are sagittal lumbar kyphosis after laminectomy and adjacent disc degeneration and pseudarthrosis after fusion. Postoperative kyphosis and iatrogenic destabilization can result in persisting pain [4]. A randomized controlled multicenter study determined whether fusion of lower lumbar spine can reduce pain and disability compared with non-surgical treatment of chronic low back pain. In that study, 294 patients with chronic lower back pain were assigned to two treatment groups (fusion or physical therapy). At the 2-year follow-up, the fusion group showed a 33\% reduction in pain, whereas the physical therapy group showed a 7\% decrease. Differences in VAS and ODI scores and return to work rates were higher in the fusion group [14].

In the current study, the outcomes of treatments in a consecutive series of patients were analyzed. The preoperative data were collected in a prospective manner, and between-group differences were not found (age, sex, hypertension, diabetes, BMI, previous surgery level, pervious surgery fusion rate, preoperative VAS score, preoperative SF-36 score, preoperative ODI score).

In the analysis of VAS pain score at 1 month, back pain VAS score increased $(0.76 \pm 1.51)$ and leg pain VAS score decreased $(-1.66 \pm 1.26)$. This result might be due to the occurrence of pain in the back muscles immediately after the operation. Given that back pain improves after 6 months and 12 months, back pain after 1 month is considered as back pain caused by back muscle damage, soft tissue irritation by fusion hardware and sacroiliac joint pain after operation rather than FBSS pain.

Significant improvement was observed at 6 and 12 months in back pain VAS score $(-0.69 \pm 1.48$ and $-1.23 \pm 2.00$, respective- ly) and in leg pain VAS score $(-2.20 \pm 1.54$ and $-2.53 \pm 1.60$, respectively). The difference between pre-treatment and posttreatment (1 year) in back pain VAS scores in the revision operation group was greater than in the radiofrequency treatment group ( -1.23 vs. -0.48$)$ but was not statistically significant $(\mathrm{p}>0.05)$. The difference between pre-treatment and post-treatment (1 year) in leg pain VAS scores in the revision operation group was also greater than in the radiofrequency treatment group ( -2.53 vs. -1.97$)$ but was not statistically significant $(\mathrm{p}>0.05)$. In addition, FBSS patients could relieve pain after the revision operation.

Regarding revision operation, in a prospective randomized controlled 1-year follow-up study of chronic low back pain after surgery for disc herniation, comparison of the effectiveness of lumbar fusion with posterior transpedicular screws and cognitive intervention with physical exercises showed that lumbar fusion failed to provide any benefit over cognitive intervention and exercises [15]. The number of additional surgeries was possibly correlated with unfavorable outcome. Extensive epidural fibrosis was associated with multiple revision and spinal instability rates that increased from $12 \%$ in the first revisional operation to $50 \%$ in the fourth revision operation [16]. The effect of revision operation for FBSS patients has been controversial. Symptoms of FBSS are either due to spinal stenosis, foraminal stenosis, mechanical instability, or facet joint pain. Among these various etiologies, radiofrequency treatment has been increasing and is reportedly effective, safe, and cost effective.

The rationale for radiofrequency is that selectively heating nervous structures can prevent nociceptive input. Percutaneous placement of small-size electrodes at target neural tissues produces different frequencies based on lesion analysis. The medial branch of the posterior primary ramus innervates the facet joints. Pain from these joints can cause recurrent back pain. Radiofrequency treatment of the medial branch of the posterior primary ramus denervates the facet joint and provides pain relief and it has proven that this is not associated with noticeable complications when provided with safety measures, especially for medial branch denervation [17].

Pulsed radiofrequency has been, however, introduced to pro- 
vide pain relief in different models without denervating the neural structures which might cause the damages or neural complication, as heat producing radiofrequency treatment for back pain. Pulsed radiofrequency has been introduced to provide pain relief in different models. Pulsed radiofrequency treatment, which modulates pain perception by affecting peripheral components of pain pathways, is applied commonly between $300 \mathrm{~Hz}$ and $300 \mathrm{GHz}$ [17-19]. Although the exact mechanism how pulsed radiofrequency causes radicular pain relief is still debatable, pulsed radiofrequency is known to disrupt neuronal membranes to produce synaptic signaling and result in electroporation [20]. Radiofrequency modulation is safe for application to lumbar dorsal root ganglion and segmental nerves. In a previous study, neurologic deficits and adverse painful side effects were not observed in 26 patients with chronic lumbosacral radicular pain refractory to conventional therapy and reportedly due to the nondestructive nature of radiofrequency [21]. Application of pulsed radiofrequency to the impaired sciatic nerve rat model resulted in radiofrequency-upregulated expression of glial cell line-derived neurotrophic factor in nerve tissue [22].

In our study, radiofrequency treatment provided pain relief and improved functional capacity and health-related quality of life in patients with pain secondary to FBSS. At 12 months, leg pain VAS score decreased from 6.3 to 4.3 and back pain VAS score decreased from 5.1 to 4.7 in the radiofrequency treatment group. The reduction in leg pain was greater than the reduction in back pain. Radiofrequency treatment can be a good choice to reduce radicular pain as well as nociceptive pain [23].

The favorable effects of radiofrequency treatment are consistent with the results in a previously reported study. Rakhmatov et al. [24] prospectively investigated 28 patients who underwent surgery for disc herniation or degenerative spinal stenosis and found pulsed radiofrequency treatment and epidural steroid administration to be effective and safe follow-up methods. The use of pulsed radiofrequency treatment was more effective than isolated epidural blockage. In a prospective study by Abejón et al. [23], the authors concluded that pulsed radiofrequency of the dorsal root ganglion was apparently less beneficial to patients with FBSS. Conversely, radiofrequency of the dorsal root ganglion was more efficacious in the herniated disc group and spinal stenosis group. Patients with FBSS had several clinical etiologies that encompassed nociceptive pain as well as neuropathic pain.

Surgical management of FBSS showed limited evidence with variable outcomes. If a patient has a surgically correctable indications (pseudoarthrosis, sagittal imbalance, discogenic pain, segmental instability, etc.), surgical clinical outcome can be improved [16]. Kim and Michelsen [25] reported successful pseudoarthrosis repair as critical for clinical success in revision surgery in FBSS patients. In their study, among FBSS patients, $81 \%$ of fused patients clinically improved, whereas only $23 \%$ of patients who failed pseudoarthrosis repair clinically improved after revision surgery. However, postoperative complication rate was higher, with wound dehiscence, deep infection, and revisional operation observed in the revision surgery group. Arts et al. [26] reported good outcome in $35 \%$ of patients, with the remaining 65\% experiencing no change or exacerbation of pain in fusion for treatment of FBSS. Revision surgery should be considered carefully because the success rate in patients with FBSS declines with each subsequent procedure. In a retrospective analysis, repeated revisional surgery was shown to increase the risk of inadvertent dural tears and neural injury; the previous surgery resulted in adhesion and scarring that renders normal anatomical landmarks difficult to discern [27].

Evidence-based guidelines for a treatment regimen of FBSS are lacking because the etiology is unclear and randomized control studies are limited.

Radiofrequency treatment is a safe and effective procedure and less invasive than surgical intervention. In our opinion, performing diagnostic nerve block with relevant imaging data is important before making decision for revision surgery. Radiofrequency treatment can be considered when patients have multiple comorbidities and are not suitable for surgery when positive response to diagnostic nerve block, especially when pain with/without sensory symptoms in patients without major neurological deficits.

The present study had several limitations. First, the study was retrospective without a randomized or control group, and effect of analgesic medication was not analyzed. However, most of treated patients had limited effect from medication and there were no patients who required more or stronger pain medications such as opioids following treatment. Second, the sample size was small, and follow-up period was short (1 year). Larger studies with longer follow-up duration are necessary in the following study to determine the long-term pain outcomes and late complications, as well as how many treated patients had undergone repeated procedures with successful results, and how many had undergone surgeries from treated group.

\section{CONCLUSION}

Compared with revision operation, radiofrequency treatment is not inferior on early clinical outcome (up to 1 year following 
the treatment) in terms of pain relief, functional capacity, patient satisfaction, or health-related quality of life in patients with FBSS. Radiofrequency treatment can be considered, when diagnostic nerve block shows positive response, for patients who are not good candidates for surgical treatment based on imaging results or underlying diseases.

\section{CONFLICTS OF INTEREST}

No potential conflict of interest relevant to this article was reported.

\section{REFERENCES}

1. Thomson S. Failed back surgery syndrome - definition, epidemiology and demographics. Br J Pain 2013;7:56-59

2. Rigoard P, Desai MJ, Taylor RS. Failed back surgery syndrome: what's in a name? A proposal to replace "FBSS" by "POPS”.... Neurochirurgie 2015;61 Suppl 1:S16-S21

3. Waguespack A, Schofferman J, Slosar P, Reynolds J. Etiology of long-term failures of lumbar spine surgery. Pain Med 2002;3:18-22

4. Assaker R, Zairi F. Failed back surgery syndrome: to re-operate or not to re-operate? A retrospective review of patient selection and failures. Neurochirurgie 2015;61 Suppl 1:S77S82

5. Baber Z, Erdek MA. Failed back surgery syndrome: current perspectives. J Pain Res 2016;9:979-987

6. Cho JH, Lee JH, Song KS, Hong JY. Neuropathic pain after spinal surgery. Asian Spine J 2017;11:642-652

7. Cho JH, Lee JH, Song KS, Hong JY, Joo YS, Lee DH, et al.. Treatment outcomes for patients with failed back surgery. Pain Physician 2017;20:E29-E43

8. Kapural L, Mekhail N. Radiofrequency ablation for chronic pain control. Curr Pain Headache Rep 2001;5:517-525

9. Cohen SP, Sireci A, Wu CL, Larkin TM, Williams KA, Hurley RW. Pulsed radiofrequency of the dorsal root ganglia is superior to pharmacotherapy or pulsed radiofrequency of the intercostal nerves in the treatment of chronic postsurgical thoracic pain. Pain Physician 2006;9:227-235

10. Delgado DA, Lambert BS, Boutris N, McCulloch PC, Robbins AB, Moreno MR, et al.. Validation of digital visual analog scale pain scoring with a traditional paper-based visual analog scale in adults. J Am Acad Orthop Surg Glob Res Rev 2018;2:e088.

11. Fairbank JC, Couper J, Davies JB, O’Brien JP. The Oswestry low back pain disability questionnaire. Physiotherapy
$1980 ; 66: 271-273$

12. Lins L, Carvalho FM. SF-36 total score as a single measure of health-related quality of life: scoping review. SAGE Open Med 2016;4:2050312116671725

13. Sebaaly A, Lahoud MJ, Rizkallah M, Kreichati G, Kharrat K. Etiology, evaluation, and treatment of failed back surgery syndrome. Asian Spine J 2018;12:574-585

14. Fritzell P, Hägg O, Wessberg P, Nordwall A; Swedish Lumbar Spine Study Group. 2001 Volvo Award Winner in Clinical Studies: lumbar fusion versus nonsurgical treatment for chronic low back pain: a multicenter randomized controlled trial from the Swedish Lumbar Spine Study Group. Spine (Phila Pa 1976) 2001;26:2521-2532; discussion 2532

15. Brox JI, Reikerås O, Nygaard $\varnothing$, Sørensen R, Indahl A, Holm I, et al.. Lumbar instrumented fusion compared with cognitive intervention and exercises in patients with chronic back pain after previous surgery for disc herniation: a prospective randomized controlled study. Pain 2006;122: 145-155

16. Skaf G, Bouclaous C, Alaraj A, Chamoun R. Clinical outcome of surgical treatment of failed back surgery syndrome. Surg Neurol 2005;64:483-488, discussion 488

17. Sluijter ME. The role of radiofrequency in failed back surgery patients. Curr Rev Pain 2000;4:49-53

18. Cahana A, Van Zundert J, Macrea L, van Kleef M, Sluijter M. Pulsed radiofrequency: current clinical and biological literature available. Pain Med 2006;7:411-423

19. Leggett LE, Soril LJ, Lorenzetti DL, Noseworthy T, Steadman R, Tiwana S, et al.. Radiofrequency ablation for chronic low back pain: a systematic review of randomized controlled trials. Pain Res Manag 2014;19:e146-e153

20. Cosman ER Jr, Cosman ER Sr. Electric and thermal field effects in tissue around radiofrequency electrodes. Pain Med 2005;6:405-424

21. Simopoulos TT, Kraemer J, Nagda JV, Aner M, Bajwa ZH. Response to pulsed and continuous radiofrequency lesioning of the dorsal root ganglion and segmental nerves in patients with chronic lumbar radicular pain. Pain Physician 2008;11:137-144

22. Jia Z, Ren H, Li Q, Ji N, Luo F. Pulsed radiofrequency reduced neuropathic pain behavior in rats associated with upregulation of GDNF expression. Pain Physician 2016;19: 49-58

23. Abejón D, Garcia-del-Valle S, Fuentes ML, Gómez-Arnau JI, Reig E, van Zundert J. Pulsed radiofrequency in lumbar radicular pain: clinical effects in various etiological groups. Pain Pract 2007;7:21-26 
24. Rakhmatov KR. Treatment of postoperative radicular pain syndrome with pulsed radiofrequency ablation of the spinal ganglia. EPRA Int J Multidiscip Res (IJMR) 2020;6:407-413

25. Kim SS, Michelsen CB. Revision surgery for failed back surgery syndrome. Spine (Phila Pa 1976) 1992;17:957-960

26. Arts MP, Kols NI, Onderwater SM, Peul WC. Clinical outcome of instrumented fusion for the treatment of failed back surgery syndrome: a case series of 100 patients. Acta Neurochir (Wien) 2012;154:1213-1217

27. Khan IS, Sonig A, Thakur JD, Bollam P, Nanda A. Perioperative complications in patients undergoing open transforaminal lumbar interbody fusion as a revision surgery. J Neurosurg Spine 2013;18:260-264 